\title{
Thin-layer hydration method to prepare a green tea extract niosomal gel and its antioxidant performance
}

Original Paper

\author{
Chasanah U.『 , Mahmintari N., Hidayah F., El Maghfiroh F.A., Rahmasari D., Weka Nugraheni R. \\ Department of Pharmacy, Faculty of Health Science, \\ University of Muhammadiyah Malang, \\ Malang 65145, Indonesia
}

Received 14 December, 2020, accepted 29 June, 2021

Abstract This study aimed to prepare a niosomal gel of green tea (Camellia sinensis) extract containing catechins, mostly epigallocatechin3-gallate (ECGC), as a potent antioxidant. Niosomes can increase EGCG's stability and penetration into the skin for a better therapeutic effect. Niosomes were prepared by a thin-layer hydration method, were evaluated for their vesicle shape, particle size, polydispersity index, zeta potential and entrapment efficiency, and then incorporated into gels using sodium alginate as a gelling agent. Three niosomal gel formulations were prepared with different concentrations of niosomes green tea extract. Afterwards, organoleptic properties, chemical and physical characteristics, antioxidant activity, and stability and irritability of the niosomal gels were investigated. The different concentrations of green tea extract had a significant effect on the physical characteristics, but not on the chemical ones. Its antioxidant activity was determined using the 1,1-diphenyl-2-picrylhydrazyl (DPPH) scavenging method. The $50 \%$ extract green tea niosomes gel showed the highest inhibition value (25.13\%). The stability was determined by freeze-thaw and real-time methods; they showed a decrease in $\mathrm{pH}$, but still within the $\mathrm{pH}$ range of skin. The irritability test used was the Hen's Egg Test-Chorioallantoic Membrane (HET-CAM) method, which showed no irritation for all formulas. In conclusion, 50\% green tea extract niosomes gel results showed it to be the best formulation with optimal antioxidant results.

Keywords green tea extract-niosomes gel-antioxidant activity-stability-irritability

\section{INTRODUCTION}

Ageing is a multifactorial process that causes structural and physiological changes in each layer of the skin, such as fine lines and wrinkles, depigmentation, pale skin colour, change in texture and the loss of elasticity (Draelos, 2016). Ageing is influenced by endogenous and exogenous factors (Ramose-Silva et al., 2013). Extrinsic skin damage can develop due to several factors, and it is thought that among the environmental factors, UV rays are usually the most damaging (Perricone, 2008). UV induces the generation of reactive oxygen species (ROS) in the skin; oxidative stress conditions develop when the ROS formation exceeds the antioxidant defence ability of the affected cells (Poljšak \& Dahmane, 2012). One strategy to reduce the risk of UV rays is the use of exogenous antioxidants, which could be administered orally or topically (Pandel et al., 2013).

Green tea (Camellia sinensis) contains bioactive chemicals and it is particularly rich in the flavanol group of polyphenols known as catechins, which may constitute up to $30 \%$ of dry leaf weight (Graham, 1992). Epigallocatechin-3-gallate
(EGCG) and epicatechin-3-gallate (ECG) are the most effective antioxidant compounds found in green tea (Namal Senanayake, 2013). EGCG is easily oxidated and unstable when heated or exposed to light (Widyaningrum et al., 2015). EGCG has a low skin-penetrating ability because of its chemical interaction with the skin lipid bilayer (Wisuitiprot et al., 2011). It is soluble in water or has high hydrophilicity, making it difficult to penetrate the cell membrane (Song et al., 2014). Therefore, it is necessary to create a delivery system that can protect EGCG from degradation as well, so that it will increase the green tea extract's effectiveness as an antioxidant.

Niosomes are vesicle systems that are amphiphilic in nature and entrap hydrophilic drugs in the core cavity and hydrophobic drugs in the non-polar region present within the bilayer; hence, both hydrophilic and hydrophobic drugs can be incorporated into the niosomes (Shakya \& Bansal, 2018). Niosomes exhibit similar physical-chemical properties to liposomes, but the bilayers are formed by nonionic

\section{*E-mail: uswatun@umm.ac.id}

O Open Access. $\odot 2021$ European Pharmaceutical Journal, published by Sciendo. (cc)BY-NC-ND This work is licensed under the Creative Commons Attribution-NonCommercialNoDerivatives 3.0 License. 
surfactants. Compared with liposomes, niosomes are highly stable for a longer period (Kumar, 2019; Bartelds et al., 2018). The vesicles can work as a depot to release the drug slowly, providing a controlled release (Sharma et al., 2018). Niosomes also increase drug stability, accelerate the therapeutic effect and increase the drug's penetration ability when applied topically (Muzzalupo \& Tavano, 2015). The bilayer structure of niosomes can protect the enclosed active pharmaceutical ingredient from heterogeneous factors, so it can be used for labile and sensitive drugs. Niosomes are suitable for encapsulating a variety of active pharmaceutical ingredients (Sankhyan \& Pawar, 2012).

Topical gels have the advantage of being easily applied, distributing evenly to the skin, easily drying, giving a cold feeling to the skin and forming a layer that is easily washed away as needed (Rathod \& Mehta, 2015). Furthermore, the physiochemical properties of niosomes are maintained (Rajkumar et al., 2019). Also, because of their high water content and swelling processes, they can offer a better feeling for the skin. Furthermore, gel preparations are suitable for drugs with hydrophilicity (Silna et al., 2016).

In this study, a topical antioxidant preparation was made with the niosomes system and incorporated into the gel with sodium alginate as the gelling agent to overcome the absorption and stability problems of EGCG in green tea extract. Alginate is widely used as a hydrogel agent in biomedicine, including drug delivery applications. It is widely used due to its biocompatibility, low toxicity, relatively low cost and mild gelation (Lee \& Mooney, 2012).

\section{MATERIALS AND METHODS}

\section{Materials}

\section{Niosome preparation}

The materials used were cholesterol (Sigma-Aldrich); sorbitan monostearate (Span 60) from CRODA Health Care; polysorbate 60 (Tween 60) from Matching Nature; calcium chloride $\left(\mathrm{CaCl}_{2}\right)$ and polyethylene glycol 400 (PEG 400) from Merck; methylparaben, sodium benzoate, propylene glycol, carboxymethyl cellulose sodium (CMC-Na) from Bratacochem; sodium alginate from Jiejing Shadong Corporation; methanol (ProAnalytics); $0.9 \%$ sodium chloride $(\mathrm{NaCl})$, distilled water, sodium lauryl sulphate (SLS) and hen's egg (for irritation testing) purchased from an authentic source; 2,2-diphenyl1-picrylhydrazyl (DPPH) (Sigma-Aldrich); green tea extract (catechin 98\%) from In Health.

\section{Green tea extract noisome characterisation}

The green tea extract niosomes were characterised based on the vesicle shape, particle size, distribution (polydispersity index $[\mathrm{Pdl}]$ ), zeta potential and entrapment efficiency. Green tea extract niosomes samples were prepared by spreading a niosomes suspension with 5\% CMC-Na on an object glass until a dry layer was formed. The composition of green tea ( $C$. sinensis) extract niosomes is reported in Table 1.

\section{Vesicle shape}

The morphological characterisation of niosomes was determined using Scanning Electron Microscopy (SEM) at the magnifications of $1000 \times, 2000 \times$ and $5000 \times$.

\section{Niosomes particle size, Pdl and zeta potential}

The particle size, Pdl and zeta potential were determined using Zetasizer Nano (Malvern Ltd.).

\section{Entrapment efficiency (EE)}

Entrapment efficiency was determined by calculating the amount of entrapped drug. As much as $100 \mathrm{mg}$ niosomes of green tea extract was hydrated with $10 \mathrm{~mL}$ of phosphate buffer saline solution and sonicated in a bath sonicator for 10 minutes. The green tea extract containing niosomal dispersion was separated by centrifugation at $2500 \mathrm{rpm}$ for 45 minutes. The clear supernatant was analysed using a UV spectrophotometer at $\lambda_{\max }$ of $273 \mathrm{~nm}$ to calculate the amount of entrapped drug. All assessments were completed in triplicate. The percentage of drug encapsulation was calculated by the following equation (Patil et al., 2017; Khan \& Irchhaiya, 2020):

$\mathrm{EE}(\%)=\frac{(C t-C f)}{C t} \times 100$

where $\mathrm{Ct}$ is the concentration of the total drug and $\mathrm{Cf}$ is the concentration of the free drug.

\section{Preparation of green tea extract niosomes gel}

To prepare the green tea extract niosomes gel, the proportions of sodium alginate (as a gelling agent), $\mathrm{CaCl}_{2}$ (water absorbing agent to improve the gel properties), propylene glycol (humectant), methylparaben (preservative) and purified water were measured by weight and they are given in Table 2 . The gel was prepared by dissolving methylparaben in propylene glycol (mixture A). Sodium alginate was crushed until smooth and put into water. After being hydrated, it was homogenised while adding $\mathrm{CaCl}_{2}$ (mixture $\mathrm{B}$ ). Mixture A was added to mixture $B$ and then stirred with a homogeniser at $1000 \mathrm{rpm}$ at room temperature until homogeneous. The niosomes were suspended until homogeneous, after which the remaining water and fragrance were added to form a consistent gel (Isnan \& Jufri, 2017). 
Table 1. Composition of green tea (C. sinensis) extract niosomes.

\begin{tabular}{|c|c|}
\hline Material & Composition \\
\hline Span 60 & 12.6 gram \\
\hline Tween 60 & $19.62 \mathrm{gram}$ \\
\hline Cholesterol & $1.74 \mathrm{gram}$ \\
\hline PEG 400 & $90.0 \mathrm{gram}$ \\
\hline Green Tea Extract & $60.0 \mathrm{gram}$ \\
\hline Sodium Benzoate & $0.6 \mathrm{gram}$ \\
\hline Distilled water & to $600 \mathrm{ml}$ \\
\hline
\end{tabular}

Table 2. The composition of green tea extract niosome gel.

\begin{tabular}{|c|c|c|c|}
\hline Material & \multicolumn{3}{|c|}{ Composition (\%) (w/w) } \\
\hline $\begin{array}{c}\text { Green tea extract } \\
\text { niosome }\end{array}$ & 30 & 40 & 50 \\
\hline Sodium alginate & 5 & 5 & 5 \\
\hline $\mathrm{CaCl}_{2}$ & 0.2 & 0.2 & 0.2 \\
\hline Propylene glycol & 10 & 10 & 10 \\
\hline Methylparaben & 0.1 & 0.1 & 0.1 \\
\hline $\begin{array}{c}\text { Green tea } \\
\text { fragrance }\end{array}$ & sufficiently & sufficiently & sufficiently \\
\hline Distilled water & to 100 & to 100 & to 100 \\
\hline
\end{tabular}

\section{Green tea extract niosomes gel evaluation}

The evaluations of the green tea extract gel with niosomes system were carried out based on the organoleptic test, physical (viscosity, spreadability) and chemical (pH) characteristics tests, physical stability tests (high temperature, room temperature, low temperature and cycling test), as well as the antioxidant and irritability tests.

\section{Organoleptic test}

The organoleptic test was descriptive, done by observing the odour, colour and consistency of the preparation visually (Sharif et al., 2017).

\section{Determination of $\mathrm{pH}$}

The $\mathrm{pH}$ measurement was determined using a $\mathrm{pH}$ meter. The $\mathrm{pH}$ meter was calibrated with a $\mathrm{pH} 7.0$ standard buffer solution before measurement. The electrodes of the $\mathrm{pH}$ meter were dipped into $10 \mathrm{~g}$ of each gel preparation (Aiyalu et al., 2016).

\section{Determination of viscosity}

The viscosity was determined using a Brookfield viscometer. A total of $100 \mathrm{~g}$ of green tea extract gel with niosomes system was put into a beaker glass; then, a size 64 spindle was installed on the viscometer and lowered until immersed into the preparation. The rotor ran at $1.5 \mathrm{rpm}$ until the viscometer showed a stable number. Then, this value was recorded and multiplied by the correction factor according to the spindle size (Singh et al., 2013).

\section{Spreadability test}

Measurements were done by placing $0.5 \mathrm{~g}$ of green tea extract gel with niosomes system in a pair of transparent glass plates. Then, different weight loads $(0,50,100,150$ and $200 \mathrm{~g})$ were placed on the top of the glass plate for 1 minute per load (Dantas et al., 2016).

\section{Real-time stability test}

The ability of the vesicles to retain the desired characteristics of the niosomes was determined by storing the niosome suspension under different conditions: $4{ }^{\circ} \mathrm{C} \pm 2{ }^{\circ} \mathrm{C}$ (refrigerator), $30^{\circ} \mathrm{C} \pm 2{ }^{\circ} \mathrm{C}$ (room temperature) and $40^{\circ} \mathrm{C} \pm 2{ }^{\circ} \mathrm{C}$ (climatic chamber) for 1 month. A total of $10 \mathrm{~g}$ of the sample was placed into vials and stored at different temperatures. Organoleptic and $\mathrm{pH}$ values of the sample were tested on the first day (1st day) just before storage and the last day of storage (30th day) (Kumar \& Dua, 2018).

\section{Accelerated stability test (freeze-thaw cycle)}

A total of $10 \mathrm{~g}$ of each sample was placed into vials and stored at $4^{\circ} \mathrm{C} \pm 2^{\circ} \mathrm{C}$ for 24 hours and then transferred to $40^{\circ} \mathrm{C} \pm 2^{\circ} \mathrm{C}$ for 24 hours (counted as one cycle). The process was repeated up to six cycles and the organoleptic and $\mathrm{pH}$ values were evaluated at the end of the cycle (WHO, 1996).

\section{Antioxidant activity test}

Antioxidant activity was measured using the DPPH scavenging method for green tea extract, green tea extract gel and green tea extract gel with the niosomes system. (1) Green tea extract: Firstly, green tea extract solutions were prepared at 5, 10, 20, 50 and 100 ppm concentrations. The observations were carried out using a UV-Vis 1240 spectrophotometer at 517-nm wavelength and using vitamin C as an antioxidant positive control. (2) Green tea extract gel and green tea extract gel with niosomes system: Three $0.5-\mathrm{g}$ samples of each gel containing 30\%, 40\% and 50\% (green tea extract/green tea extract niosomes) were dissolved in water until $10 \mathrm{~mL}$ and stirred using ultrasonic stirring for 10 minutes. Then, the samples were dissolved in methanol until $100 \mathrm{~mL}$ concentration was reached and stirred with ultrasonic stirring for 20 minutes. The solutions were incubated for 30 minutes at $37{ }^{\circ} \mathrm{C}$. The absorbance was measured using a UV-Vis spectrophotometer 1240 at 515-517 nm wavelength (Gane \& Parki, 1958). \% Antioxidant activity was calculated by using the equation as follows: 
Table 3. The characteristics of green tea extract niosomes obtained from Malvern Zetasizer Nano.

\begin{tabular}{|c|c|c|c|c|}
\hline Niosome & Z-Average diam. $(\mathbf{n m})$ & Polydispersity Index & Zeta potential (mV) & $\begin{array}{c}\text { Entrapment } \\
\text { efficiency (\%) }\end{array}$ \\
\hline Mean & 1109 & 0.856 & -0.616 & 89.74 \\
\hline $\begin{array}{c}\text { Standard } \\
\text { Deviation }\end{array}$ & \pm 63 & \pm 0.057 & \pm 0.068 & \pm 2.52 \\
\hline
\end{tabular}
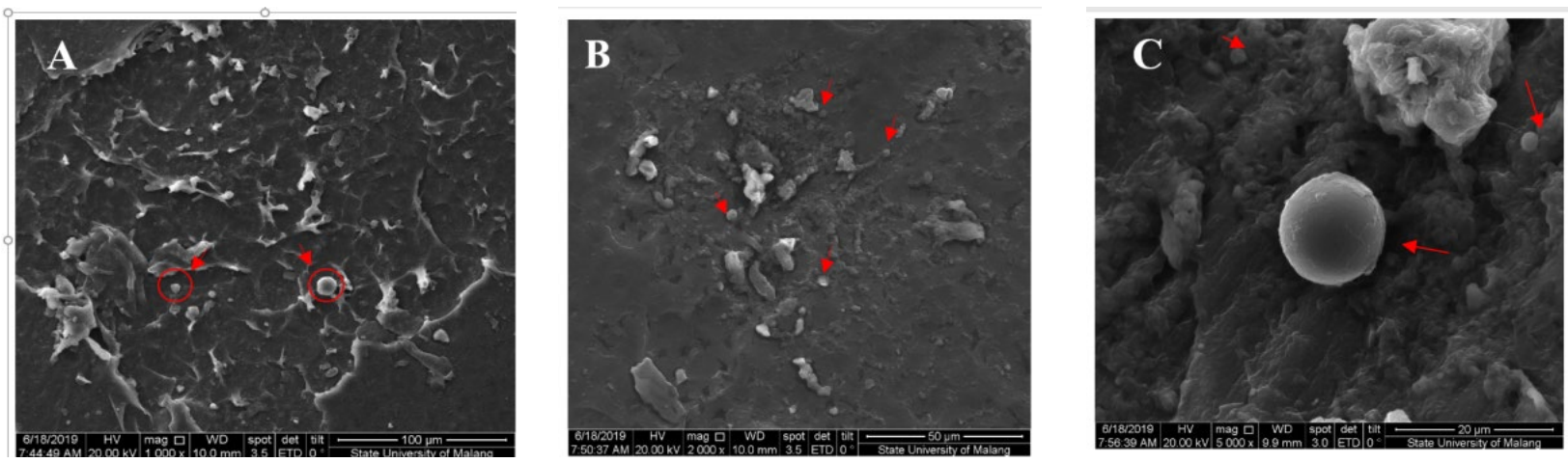

Figure 1. Scanning electron micrographs of the morphology of green tea extract niosome system at a magnification of (A) 1000x; (B) $2000 \times$ and (C) 5000x.

$\%$ antioxidant activity $=\left(A_{0}-A_{1}\right) / A_{0} \times 100$

where $A_{0}$ is the absorbance of free radical (DPPH solution) and $A_{1}$ is the absorbance of the antioxidant (sample).

\section{Irritability test}

Irritability was tested using the Hen's Egg Test Chorioallantoic Membrane (HET-CAM) method. White Leghorn eggs were prepared, put in incubator trays at $37^{\circ} \mathrm{C}$ and then rotated for 10 days. On the 10th day of incubation, the eggshell was softened by sterile $0.9 \% \mathrm{NaCl}$ and scratched around with sterile scissors. The outer membrane was moistened with a warm $0.9 \% \mathrm{NaCl}$ solution and they were put again in the incubator trays for 5-20 minutes; then, the inner egg membranes were removed to expose the vascular Chorioallantoic Membrane (CAM). After application of the test substance, about $300 \mathrm{~g}$ sample (niosomes gel of green tea extract) was put into CAM for 20 seconds and then cleaned up immediately using sterile $0.9 \%$ $\mathrm{NaCl}$ solution. The observations were started after the CAM was cleaned; the samples were examined for about 300 seconds and then scored for irritant effects (lysis, haemorrhages and coagulation). SLS was added as a positive control and distilled water as a negative control (Luepke, 1985).

\section{Statistical analysis}

The data of $\mathrm{pH}$, viscosity and spreadability test were analysed using the Shapiro-Wilk test. A $p$-value $>0.05$ indicated a normal distribution, allowing the analysis to continue with a one-way analysis of variance (ANOVA) to find a significant difference between the formulas. The test results continued with the Tukey's Honestly Significant Difference (Tukey HSD) test with a confidence level of $95 \%(a=0.05)$. If the $\mathrm{pH}$, viscosity and spreadability test had a $p$-value $<0.05$, the variance test was performed with the Kruskal-Wallis test. These analyses were performed using the Statistical Package for the Social Sciences (SPPS) v16.0 program (Chicago, USA).

\section{RESULTS AND DISCUSSION}

\section{Niosomes characterisation}

The green tea extract niosomes characteristics were observed, including the niosomes morphology (vesicle shape), particle size, distribution (Pdl), zeta potential and entrapment efficiency. The morphology is shown in Fig. 1 and the characteristics are given in Table 3.

SEM observation of niosomes at 5000× magnification showed that the niosomes were round in shape (spherical) with a smooth surface and a visible vesicle. Using the Malvern Zetasizer Nano, it was found that the average size of the majority of the green tea extract niosomes vesicles was 1109 $\mathrm{nm}$ or $1.109 \mu \mathrm{m}$ and the particle distribution showed a Pdl of 0.856 (Table 3 ). These niosomes can be classified as large unilamellar vesicles because the size of the vesicles was more than $0.10 \mu \mathrm{m}$ (Sudheer \& Kaushik, 2015). It was also found that the particle size distribution of green tea extract niosomes was very broad, because they had a Pdl value $>0.7$ (Nidhin et al., 2008). 

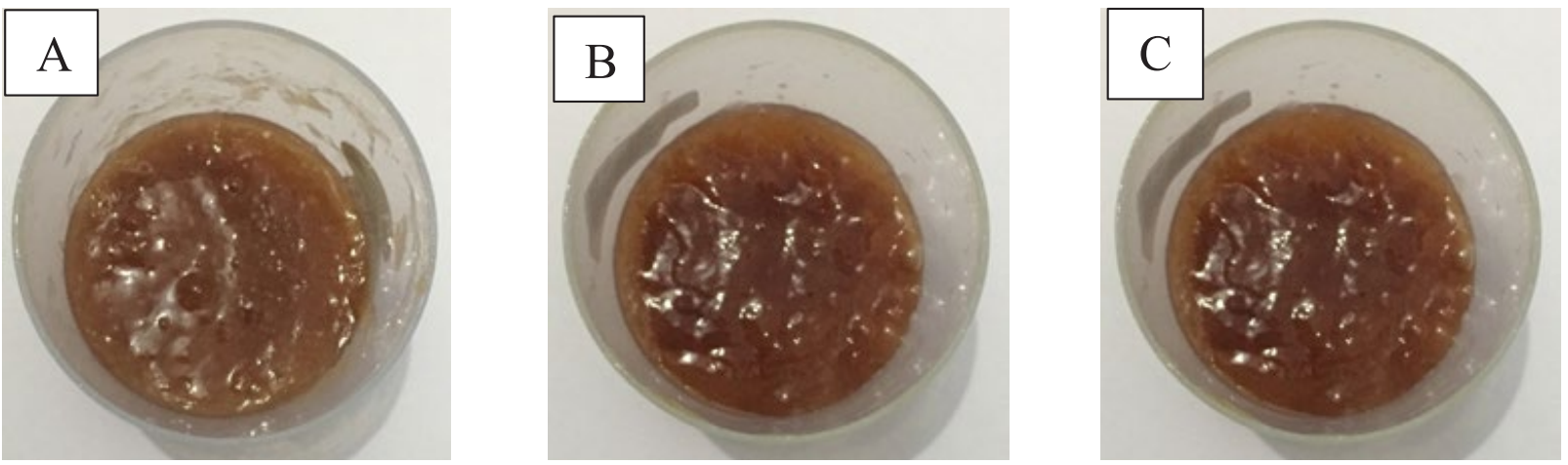

Figure 2. The organoleptic of niosome gel preparations at a niosome concentration of (A) $30 \%$, (B) $40 \%$, and (C) $50 \%$.

The niosomal suspension had a zeta potential of -0.616 $\mathrm{mV}$. This was in the range of -30 to $+30 \mathrm{mV}$, so it showed that the niosomes of green tea extract were capable of fast coagulation or flocculation (Sreeram et al., 2008; Okore et al., 2011). The result of entrapment efficiency determination given in Table 3 indicates that the green tea extract niosomes had a good entrapment efficiency with a value of $>80 \%$ (Annisa et al., 2016). This might be due to the presence of Span 60, which has the best entrapment efficiency compared with the other grades of Span (Patil et al., 2017; Khan \& Irchhaiya, 2020; Asthana et al., 2016; Jaiswal et al., 2016). It can be explained by the structure, orientation and packing behaviour of the surfactant. Span 60 has the longest saturated alkyl chain that increases the entrapment efficiency. Span 60 also has a solid nature, high phase transition temperature and high lipophilicity, which provides a less leaky system and high entrapment capability (Khan \& Irchhaiya, 2020; Jaiswal et al., 2016). The presence of cholesterol also makes the niosomes more stable (Patil et al., 2017). Increasing the cholesterol content will increase the hydrophobicity and stability of bilayer vesicles and decrease the permeability (Asthana et al., 2016). The system will be more intact and ordered as a barrier for drug release and also decrease drug leakage by improving the fluidity of the bilayer membrane (Jaiswal et al., 2016).

This study was carried out to experimentally determine the effect of various levels of green tea extract niosomes on drug delivery system characteristics. Niosomes with Span 60, Tween 60, PEG 400 and cholesterol as the main ingredients were made using the thin-film hydration method. The combination of Tween 60 and Span 60 for niosomes formulations can increase the efficiency of drug absorption and release because Tween 60 and Span 60 have chains that interact together (become entangled) and lead to better absorption (Naderinezhad et al., 2017). PEG 400 was added to help to regulate the niosomes system, so that the hydrophilicity and hydrophobicity of the system could be maintained at a balance that positively affects the absorption rate. Low concentrations of PEG 400 cause almost all the PEG 400 molecules to interact with the Span molecule through hydrogen bonds and hydrophilic action to form the amphiphilic bilayers from proteins (Hua \& Liu, 2007). PEGcoated vesicles trap some drugs in long PEG chains, thereby reducing the tendency for particle size to increase (Karin et al., 2010). Also, PEG reduces aggregation, increase stability in vivo and in vitro, and increases the entrapment efficiency and the transformation temperature (Tc) (Hua \& Liu, 2007). The addition of cholesterol molecules to the niosomes formula makes the membrane stiffer and reduces the leak of the active ingredients from the niosomes system (Karin et al., 2010).

\section{Organoleptic test}

The green tea extract gel with the niosomes system had a thick consistency, was brown in colour and had a distinctive fragrance of green tea. The higher the niosomes level, the stronger was the intensity of the brown colour as shown in Fig. 2.

\section{Physical and chemical characteristics}

The results of viscosity, $\mathrm{pH}$ value and spreadability of the green tea extract gel preparations with the niosomes system are shown in Table 4.

Different levels of green tea extract niosomes (30\%, 40\% and $50 \%$ ) had different viscosities. As can be seen in Table 4, the higher the concentration of green tea extract niosomes, the higher was the viscosity of the prepared gel. The $\mathrm{pH}$ was not significantly different and was within the $\mathrm{pH}$ range of skin tolerance (4.0-7.0) (Lambers et al., 2006). The spreadability of $30 \%$ green tea extract niosomes gel was the highest, which was $0.0051 \mathrm{~g} / \mathrm{cm}$; it was lower in the $40 \%$ green tea extract niosomes gel $(0.050 \mathrm{~g} / \mathrm{cm})$, and $50 \%$ green tea extract niosomes gel showed the lowest value $(0.0040 \mathrm{~g} / \mathrm{cm})$. This is consistent with the theory that the greater the viscosity, the lower is the spreadability (Deuschle et al., 2015). Rheological properties of green tea extract niosomes gel 30\%, 40\% and $50 \%$ were evaluated using the data from various speeds of viscosity. The rheogram (Fig. 3) showed a typical concave curve, which indicated that the system has a pseudoplastic flow. 
Table 4. Physical and chemical characteristics of green tea extract niosome gel preparation.

\begin{tabular}{|c|c|c|c|}
\hline Formula & $\begin{array}{c}\text { Viscosity } \\
\text { (Cps) }\end{array}$ & pH & $\begin{array}{c}\text { Spreadability } \\
\text { (g/cm) }\end{array}$ \\
\hline Green tea niosome gel 30\% & $292.667 \pm 1.155$ & $6.68 \pm 0.03$ & $0.0051 \pm 0.0004$ \\
\hline Green tea niosome gel 40\% & $297.333 \pm 1.115$ & $6.59 \pm 0.10$ & $0.0050 \pm 0.0003$ \\
\hline Green tea niosome gel 50\% & $306.667 \pm 2.309$ & $6.68 \pm 0.01$ & $0.0040 \pm 0.0004$ \\
\hline
\end{tabular}
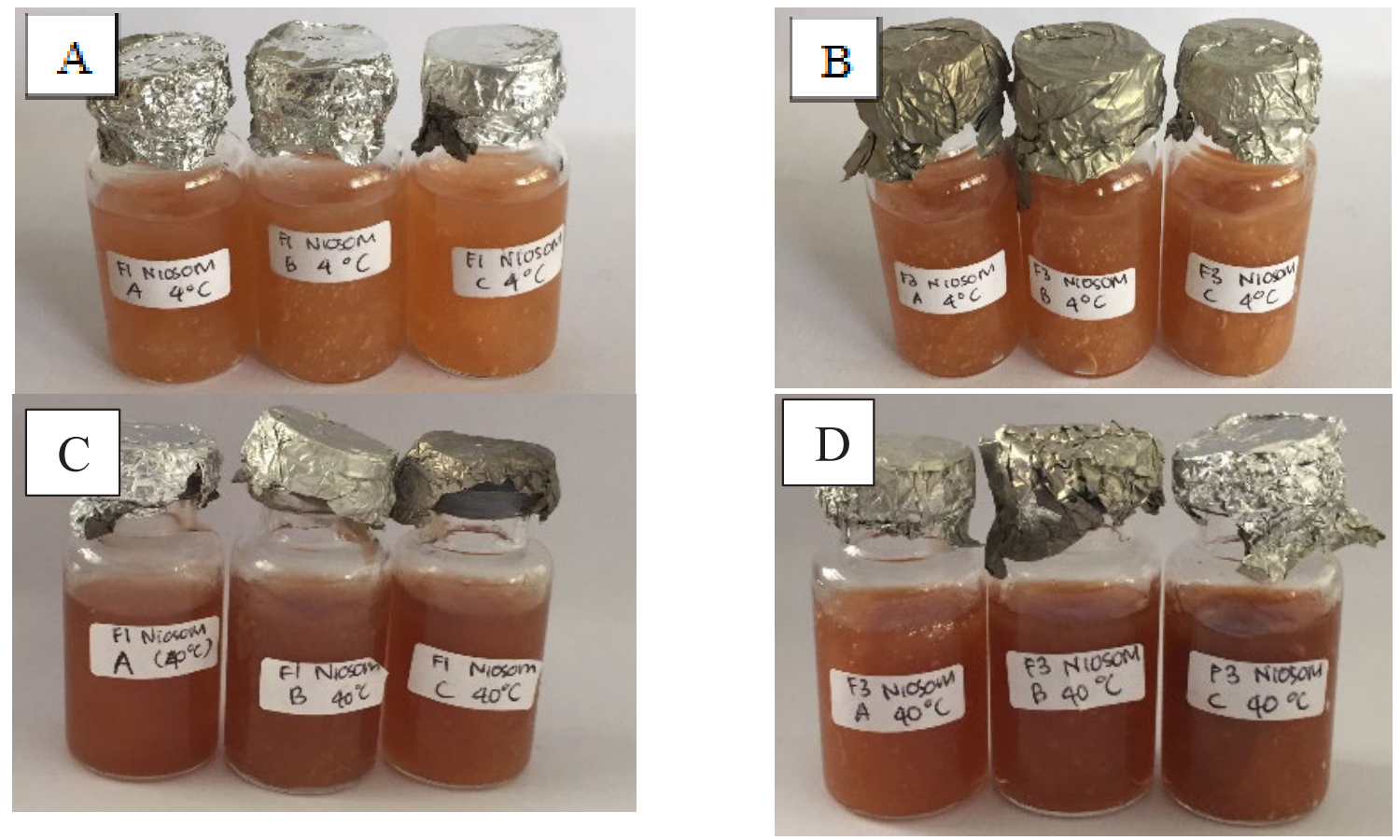

Figure 3. The organoleptic of niosome gel preparation at $4^{\circ} \mathrm{C}$ on (A) day 1 and (B) day $30 ;$ at $40^{\circ} \mathrm{C}$ on (C) day 1 and (D) day 30 shown the same consistency, brown color and distinctive fragrance of green tea as before storage.

The requirements for a gel base are that it must be inert, safe and not interact with the other ingredients of the formula. The type and concentration of different additives and extracts in the formula will affect the physical and chemical characteristics, stability, effectivity and safety level of the preparation. The base gel to carry the green tea extract niosomes system was sodium alginate. Sodium alginate was chosen because when it is combined with $\mathrm{CaCl}_{2}$, it will form a gel without any heating, is solid during the heating process, has a good physical appearance and also has abundant functional groups for a high absorption efficiency (Brovchenko et al., 2005). The function of the PEG 400 additive in green tea extract niosomes sodium alginate during the gel phase was to strengthen the gel structure bonding to form a stronger gel matrix due to increase in viscosity level.

The results of TEM (Transmission Electron Microscope) observations showed spherical niosomes morphology with a smooth surface and a diameter of $1109 \pm 63 \mathrm{~nm}$ (Table 3). Particle morphology is important because less-spherical particles will facilitate contact between the particles and could lead to aggregation. Niosomes in this study were classified as large vesicles because they were larger than 0.10 $\mu \mathrm{m}$ (Sudheer \& Kaushik, 2015). It is a bigger vesicle when compared to the previous study (Isnan \& Jufri, 2017).

The viscosity test results presented in Table 4 show that the viscosity value of this preparation did not comply with Indonesia National Standards (SNI). According to SNI-164399-1996, a good viscosity for the gel is $2.000-50.000$ cps. However, these results are similar to those of a previous study; the viscosity was very large due to the addition of gelling agent (sodium alginate) and water absorbing agent $\left(\mathrm{CaCl}_{2}\right)$. The $\mathrm{pH}$ values of green tea extract niosomes gel formula of different concentrations (30\%, 40\% and 50\%) right after manufacture were acceptable because they were within the $\mathrm{pH}$ range of topical preparations $(\mathrm{pH} 4-8)$ as stipulated in SNI16-4399-1996. So, it has a safe level of acidity for use on the skin. These $\mathrm{pH}$ values are the same as reported in a previous study (Isnan \& Jufri, 2017).

To determine the uniformity level of particle size distribution, the Pdl was measured. The good particle size distribution was 


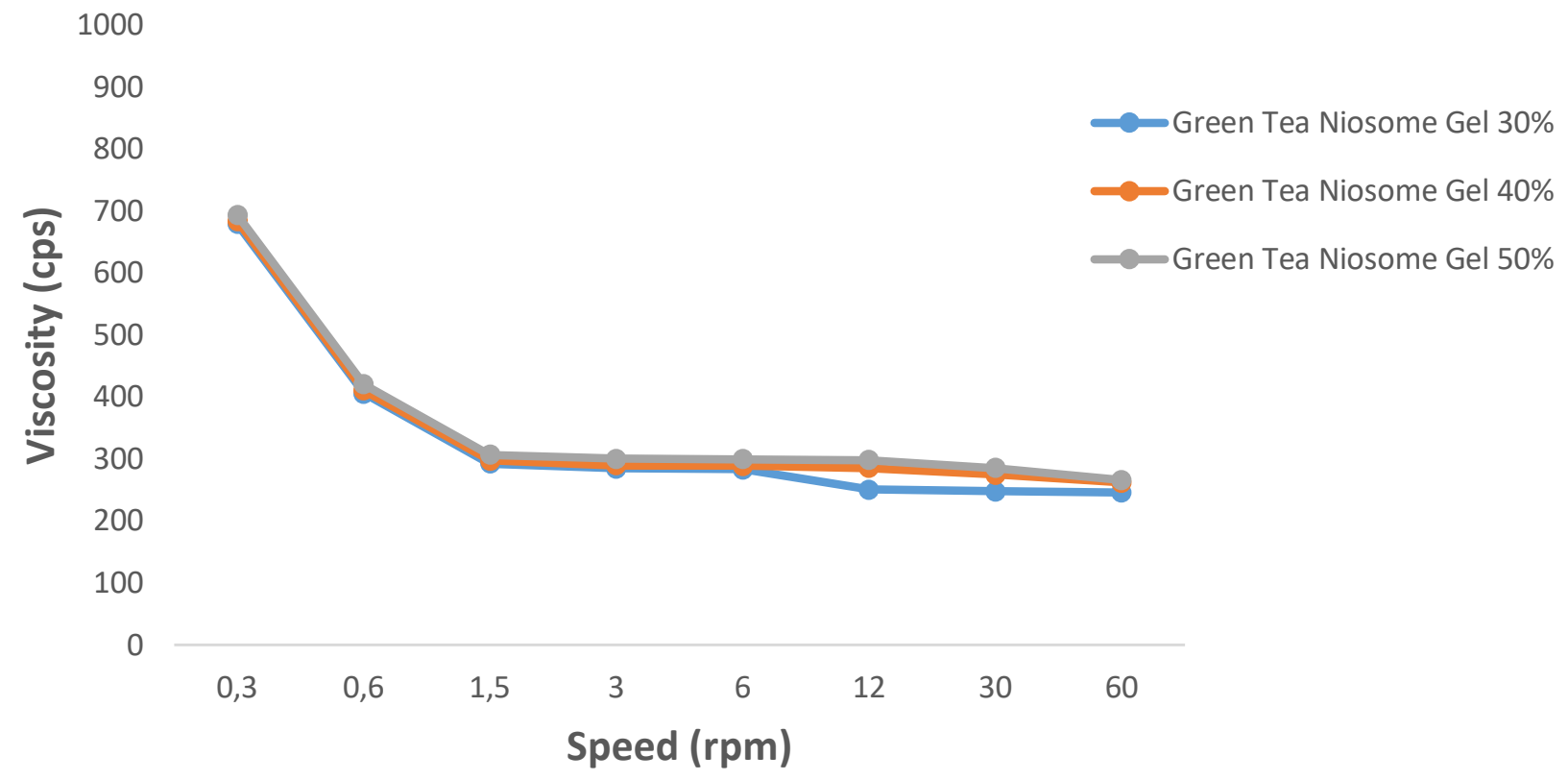

Figure 4. Rheological behavior of green tea extract niosome gel preparation.

Table 5. The $\mathrm{pH}$ value of green tea extract niosome gel preparations.

\begin{tabular}{|c|c|c|c|c|c|}
\hline Fomula & $\mathbf{1}^{\text {st }}$ day & Freeze-thaw & $\mathbf{4}^{\circ} \mathbf{C}$ & $\mathbf{3} \mathbf{0}^{\circ} \mathbf{C}$ & $\mathbf{4 0}^{\circ} \mathbf{C}$ \\
\hline $\begin{array}{l}\text { Green tea extract } \\
\text { niosome gel 30\% }\end{array}$ & $6.68 \pm 0.03$ & $6.16 \pm 0.06$ & $6.52 \pm 0.02$ & $6.02 \pm 0.19$ & $6.01 \pm 0.03$ \\
\hline $\begin{array}{c}\text { Green tea extract } \\
\text { niosome gel 40\% }\end{array}$ & $6.59 \pm 0.10$ & $6.17 \pm 0,02$ & $6.52 \pm 0.03$ & $6.06 \pm 0.04$ & $6.02 \pm 0.02$ \\
\hline $\begin{array}{c}\text { Green tea extract } \\
\text { niosome gel 50\% }\end{array}$ & $6.68 \pm 0.01$ & $6.18 \pm 0,10$ & $6.50 \pm 0.07$ & $6.04 \pm 0.10$ & $5.96 \pm 0.06$ \\
\hline
\end{tabular}

$\leq 0.3$ which shows a homogenous population for colloidal system (Seleci et al., 2016). Particles can be classified into monodispersion groups (Pdl value <0.7) and large size distributions (Pdl values >0.7) (Okore et al., 2011). The niosomes $\mathrm{Pdl}$ in this study showed an uneven uniformity of particle size because the $P d l$ value was $\geq 0.3$. This result was contrary to that of a previous study which showed a Pdl value of 0.349 (Isnan \& Jufri, 2017).

\section{Green tea extract niosomes gel stability test}

\section{Real-time stability test}

It was found that $30 \%, 40 \%$ and $50 \%$ green tea extract niosomes gels were the most stable when stored at $4{ }^{\circ} \mathrm{C}$. However, the overall result of organoleptic observations showed that $30 \%$, $40 \%$ and $50 \%$ green tea extract niosomes gel did not undergo changes in colour, odour and consistency after storage for 30 days and they showed the same organoleptic characteristics as on day 1 (before storage), as can be observed in Fig. 4. Furthermore, the $\mathrm{pH}$ values of the gel preparations stored at $4{ }^{\circ} \mathrm{C}, 30^{\circ} \mathrm{C}$ and $40{ }^{\circ} \mathrm{C}$ were decreased slightly over time, as shown in Table 5.

This finding can be due to the low stability of niosomes after manufacture, as shown by the zeta potential value. A sample is called 'stable' if it has a zeta potential more positive than $+30 \mathrm{mV}$ or more negative than $-30 \mathrm{mV}$ (Surini et al., 2018). The zeta potential of the green tea extract niosomes sample was $-0.616 \mathrm{mV}$ (Table 3), which means that the green tea extract niosomes preparation is less stable than found in a previous study in which it was reported as $-39.9 \mathrm{mV}$ (Isnan \& Jufri, 2017). The zeta potential of green tea extract niosomes was low or close to zero because the constituent ingredients were Tween 60 and Span 60, which are nonionic surfactants. To ensure the preparations retain the same properties after manufacture, the physicochemical characteristics and stability tests were carried out on the green tea extract niosomes gel preparations. The difference between the three formulas was in the colour intensity because green tea extract has a brown colour; the higher the levels of niosomes in the gel base, the browner the colour. Gel preparations had a distinctive smell of green tea because the fragrance of green 
tea was used in them. The gel preparation also had a thick consistency as $5 \%$ sodium alginate was used as the gelling agent in them. These results are similar to those of a previous study, but the colour was darker due to the increased use of green tea extract (Isnan \& Jufri, 2017).

\section{Accelerated stability test}

Accelerated stability test using a freeze-thaw cycling test method showed that all the formulas did not change organoleptically. All gels were brown, remained with their typical green tea flavour and had a thick consistency. The $\mathrm{pH}$ value measurement results of the freeze-thaw cycling test are shown in Table 5. The three formulas were not significantly different. However, on comparing them to the initial $\mathrm{pH}$ values of the preparation before the stability test, the $\mathrm{pH}$ values of all gels were found to be decreased.

Niosomal gel stability evaluation showed that the preparation was most stable after 30 days when stored at a low temperature $\left(4{ }^{\circ} \mathrm{C}\right)$, because the reaction slowed at that temperature. But overall, there were no significant changes in $\mathrm{pH}$ value, no changes in odour or colour and no visible syneresis, as seen with both the real-time stability and freeze-thaw stability methods (Fig. 4). Overall, this showed that the niosomes system successfully gives a better encapsulation for the active ingredient and increases the stability of preparations.

\section{Antioxidant activity}

Before being added to the niosomes system, the green tea extract was examined for its antioxidant activity using the DPPH scavenging method. It was found that the antioxidant activity of green tea extract had an Inhibitory Concentration $50 \%$ (IC50) value of $45.56 \mu \mathrm{g} / \mathrm{mL}$, while the IC50 of vitamin C, used as a positive control, was $5.31 \mu \mathrm{g} / \mathrm{mL}$. The extract is classified as a very strong antioxidant because it has an IC50 lower than $50 \mu \mathrm{g} / \mathrm{mL}$ (Gane \& Parki, 1958). The results of the antioxidant activity of the green tea extract gels and green tea extract niosomes gels are presented in Tables 6 and 7 .

The inhibition percentage was obtained from the measurement of $1000 \mu \mathrm{g} / \mathrm{mL}$ samples. The inhibition of $30 \%$ green tea extract gel was $17.78 \%$ and that of its green tea extract niosomes gel was $10.04 \%$; the $40 \%$ green tea extract gel had an inhibition of $19.69 \%$ and its green tea extract niosomes gel had a value of $17.49 \%$; the $50 \%$ green tea extract gel had an inhibition of $27.09 \%$ and its green tea extract niosomes gel had a value of $25.13 \%$. The antioxidant activity increases along with increasing levels of niosomes green tea extract. But it also implies that the addition of green tea extract into niosomes gel has less antioxidant activity compared to conventional green tea extract gel. Besides, the antioxidant activity of green tea extract gel and green tea extract niosomes gel was increased as the concentration of green tea extract niosomes increased (Tables 6 and 7). This finding shows that the niosomes system
Table 6. Antioxidant activity of green tea extract gel.

\begin{tabular}{|c|c|}
\hline Formula & \% inhibition \\
\hline Green tea extract gel $30 \%$ & $17.78 \pm 1.49$ \\
\hline Green tea extract gel $40 \%$ & $19.69 \pm 1.14$ \\
\hline Green tea extract gel $50 \%$ & $27.09 \pm 1.57$ \\
\hline
\end{tabular}

Table 7. Antioxidant activity of green tea extract niosome gel.

\begin{tabular}{|c|c|}
\hline Formula & \% inhibition \\
\hline Green tea extract niosome gel 30\% & $10.04 \pm 0.05$ \\
\hline Green tea extract niosome gel 40\% & $17.49 \pm 0.03$ \\
\hline Green tea extract niosome gel 50\% & $25.13 \pm 0.05$ \\
\hline
\end{tabular}

provides a high entrapment for active ingredients and its effectiveness as an antioxidant can be decreased due to the ability of the active ingredient to release from the niosomes gel matrix. This study showed a better entrapment compared to the previous study, which reported $77.80 \%$ as the highest entrapment efficiency (Isnan \& Jufri, 2017).

\section{Irritability test}

The results of the irritability test are shown in Table 8. SLS as a positive control caused the CAM to haemorrhage in 48 seconds, lysis in 120 seconds and coagulation in 228 seconds. The irritation score was 10.63 , which is classified as the strongly irritating category. Distilled water as a negative control caused no haemorrhage, lysis or coagulation on the CAM, which could be classified as the no irritation category. The green tea extract niosomes gel with various levels of green tea extract niosomes showed 0 irritation scores as well, which indicates that the green tea extract niosomes gel did not irritate whatsoever (Luepke, 1985).

Lastly, the irritation test results showed that the green tea extract niosomes gel caused no sign of irritation (haemorrhage, lysis and coagulation) on the chorioallantoic membrane of hen's eggs (Table 8). The niosomes system gave a good entrapment of the active ingredient, which led to better interaction between the green tea extract niosomes gel and the chorioallantoic membrane. No irritation test was conducted in the previous studies of niosomes green tea extract. However, safety testing is essential before raw materials or end products can be sold to customers (Yanti Eff et al., 2019). This test provides practical information on the potential skin damages caused by these topical green tea extract niosomes gel (Suksaeree and Chuchote, 2018).

\section{CONCLUSION}

The 30\% green tea extract niosomes gel had the best physical properties, showing the highest $\mathrm{pH}$ value, viscosity 
Table 8. Irritation test results of green tea extract niosome gel

\begin{tabular}{|c|c|c|c|}
\hline \multirow{2}{*}{ Formula } & \multicolumn{2}{|c|}{ Treatment } & \multirow{2}{*}{ Irritation Score } \\
\hline & Before & After & \\
\hline SLS (positive control) & & & 10.63 (Strong irritation) \\
\hline Aquadest (negative control) & & & 0 (No irritation) \\
\hline Green tea extract niosome gel $30 \%$ & & & 0 (No irritation) \\
\hline Green tea extract niosome gel $40 \%$ & & & 0 (No irritation) \\
\hline Green tea extract niosome gel 50\% & & & 0 (No irritation) \\
\hline
\end{tabular}

and spreadability on human skin. All formulations showed good stability with no change in their organoleptic properties. The $50 \%$ green tea extract niosomes gel had the greatest antioxidant activity. Lastly, all formulas also showed no irritation to the hen's egg membrane. The results demonstrated that variation in the concentration of green tea extract niosomes affected the physicochemical characteristics and antioxidant activity, but did not affect the irritability test. We suggest additional evaluation and continuing the stability testing for up to 90 days to improve the preparation of green tea extract niosomes gel. The release of active compounds and penetration evaluation should also be studied to determine the optimum quality of niosomes system.

\section{CONFLICT OF INTEREST}

The authors declare no conflict of interest.

\section{ACKNOWLEDGEMENT}

The authors thank the Directorate Research and Community Service, University of Muhammadiyah Malang for supporting this research. 
[1] Aiyalu R, Govindarjan A, Ramasamy A. Formulation and evaluation of topical herbal gel for the treatment of arthritis in animal model. Brazilian J Pharm Sci. 2016;52:493-507.

[2] Annisa R, Hendradi E, Melani D. Pengembangan sistem nanostructured lipid carriers (NIC) meloxicam dengan lipid monostearin dan miglyol 808 menggunakan metode emulsifikasi [Development of the nanostructured lipid carrier (NIC) meloxicam system with monostearin and miglyol 808 lipids using the emulsification method]. J Trop Pharm Chem. 2016;3:156-169.

[3] Asthana GS, Sharma PK, Asthana A. In vitro and in vivo evaluation of niosomal formulation for controlled delivery of clarithromycin. Scientifica (Cairo). 2016:1-10.

[4] Bartelds R, Nematollahi M, Pols T, et al. Niosomes, an alternative for liposomal delivery. PLoS One. 2018:1-18.

[5] Brovchenko I, Krukau A, Smolin N, Oleinikova A, Geiger A, Winter R. Cross-linking polymers - alginate worms. RSC Adv Chem Sci. 2005;59:3-5

[6] Dantas MGB, Reis SAGB, Damasceno C, et al. Development and evaluation of stability of a gel formulation containing the monoterpene borneol. Sci World J. 2016;2016:1-4.

[7] Deuschle VCKN, Deuschle RAN, Bortoluzzi MR, Athayde ML. Physical chemistry evaluation of stability, spreadability, in vitro antioxidant, and photo-protective capacities of topical formulations containing calendula officinalis L. Leaf extract. Brazilian J Pharm Sci. 2015;51:63-75.

[8] Draelos Z. Cosmetic Dermatology. New Jersey: Wiley Blackwell; 2016.

[9] Gane N, Parki. Antioxidant determination by the use of a stable free radical. Nat Microbiol. 1958;182:1199-1200.

[10] Graham HN. Green tea composition, consumption, and polyphenol chemistry. Prev Med (Baltim). 1992;21:334-350.

[11] Hua W, Liu T. Preparation and properties of highly stable innocuous niosomes in Span 80/PEG 400/H2O system. Colloids Surfaces A Physicochem. Engin Aspects. 2007;302:377-382.

[12] Isnan AP, Jufri M. Formulation of niosomal gel containing green tea extract (Camellia sinensis L. Kuntze) using thin-layer hydration. Int J Appl Pharm. 2017;9:38-43.

[13] Jaiswal PH, Gujarathi NA, Rane BR, Pawar SP. Formulation of niosomal gel of diclofenac sodium and its in-vitro characterization. Int J Pharm Pharm Res. 2016;64:585-600.

[14] Junyaprasert VB, Singhsa P, Suksiriworapong J, Chantasart D. Physicochemical properties and skin permeation of Span 60/ Tween 60 niosomes of ellagic acid. Int J Pharm. 2012;423:303-311

[15] Lee Ky, Mooney DJ. Alginate: Properties and biomedical applications. Prog Polym Sci. 2012;37:106-126.

[16] Karim KM, Mandal AS, Biswas N, et al. Niosomes: A future of targeted drug delivery systems. J Adv Pharm Technol Res. 2010;1:374-380.

[17] Khan R, Irchhaiya R. In vitro in vivo evaluation of niosomal formulation of famotidine. Int J Pharm Pharm Sci. 2020;12:15-22.

[18] Kumar A, Dua JS. Formulation and evaluation of itraconazole niosomal gel. Asian J Pharm Res Dev. 2018;6:76-80.
[19] Kumar R. Lipid-Based Nanoparticles for Drug-Delivery Systems. New York: Elsevier Inc; 2019.

[20] Lambers H, Piessens S, Bloem A, Pronk H, Finkel P. Natural skin surface $\mathrm{pH}$ is on average below 5 , which is beneficial for its resident flora. Int J Cosmet Sci. 2006;28:359-370.

[21] Luepke NP. Hen's egg chorioallantoic membrane test for irritation potential. Food Chem Toxicol. 1985;23:287-291.

[22] Mohd Nadzir M, Tan WF, Mohamed AR, Hisham SF. Size and stability of curcumin niosomes from combinations of tween 80 and span 80. Sains Malaysiana. 2017;46:2455-2460.

[23] Muzzalupo R, Tavano L. Niosomal drug delivery for transdermal targeting: Recent advances. Res Reports Transdermal Drug Deliv. 2015;4:23-33.

[24] Naderinezhad S, Amoabediny G, Haghiralsadat F. Co-delivery of hydrophilic and hydrophobic anticancer drugs using biocompatible $\mathrm{pH}$-sensitive lipid-based nano-carriers for multidrug-resistant cancers. RSC Adv. 2017;7:30008-30019.

[25] Namal Senanayake SPJ. Green tea extract: Chemistry, antioxidant properties and food applications - A review. J Funct Foods. 2013;5:1529-1541.

[26] Nidhin M, Indumathy R, Sreeram KJ, Nair BU. Synthesis of iron oxide nanoparticles of narrow size distribution on polysaccharide templates. Bull Mater Sci. 2008;31:93-96.

[27] Okore VC, Attama AA, Ofokansi KC, Esimone CO, Onuigbo EB. Formulation and evaluation of niosomes. Indian J Pharm Sci. 2011;73:323-328.

[28] Pandel R, Poljšak B, Godic A, Dahmane R. Skin photoaging and the role of antioxidants in its prevention. ISRN Dermatol. 2013;2013:1-11.

[29] Patil VB, Tadavi SA, Pawar SP. Formulation of niosomal gel of aceclofenac and its in-vitro characterization. Int J Pharma Chem Res. 2017;3:467-473.

[30] Perricone N. Skin aging. Acta Derm Venereol. 2008;17:47-53.

[31] Poljšak B, Dahmane R. Free radicals and extrinsic skin aging. Dermatol Res Pract. 2012;2012:1-4.

[32] Rajkumar J, GV R, Sastri KT, Burada S. Recent update on proniosomal gel as topical drug delivery system. Asian J Pharm Clin Res. 2019;12:54-61.

[33] Ramos-e-Silva L, Celem, MR, Ramos-e-Silva S, Fucci-da-Costa AP. Anti-aging cosmetics: Facts and controversies. Clin Dermatol. 2013;31:750-758.

[34] Rathod HJ, Mehta DP. A review on pharmaceutical gel. Acta Sci Int J Pharm Sci. 2019;1:33-47.

[35] Sankhyan A, Pawar P. Recent trends in niosomes as vesicular drug delivery system. J Appl Pharm Sci. 2012;2:20-32.

[36] Seleci DA, Seleci M, Walter JG, Stahl F, Scheper T. Niosomes as nanoparticular drug carriers: Fundamentals and recent applications. J Nanomater. 2016;2016:1-13.

[37] Shakya V, Bansal BK. Niosomes: a novel trend in drug delivery. Int J Res Dev Pharm Life Sci. 2018;11:5205

[38] Sharif MK, Sharif HR, Nasir M. Sensory evaluation and consumer acceptability. In: Handbook of Food Science and Technology, Chapter: Sensory Evaluation and Consumer Acceptability. 2017:362-286. 
[39] Sharma D, Ali AAE, Aate JR. Niosomes as novel drug delivery system: Review article. PharmaTutor. 2018;6:58-65.

[40] Silna EA, Krishnakumar K, Nair SK, Narayanan VA. Hydrogels in topical drug delivery - A review. Int J Innov Drug Discov. 2016;6:87-93.

[41] Singh VK, Singh PK, Sharma PK, Srivastava PK, Mishra A. Formulation and evaluation of topical gel of acelofenac containing piparine. Indo Am J Pharm Res. 2013;3:5266-5280.

[42] Song $Q$, Li D, Zhou $Y$, et al. Enhanced uptake and transport of (+)-catechin and (-)-epigallocatechin gallate in niosomal formulation by human intestinal C aco-2 cells. Int J Nanomedicine. 2014;9:2157-2165.

[43] Sudheer P, Kaushik K. Review on niosomes - a novel approach for drug targeting. J Pharm Res. 2015;14:20-25.

[44] Suksaeree J, Chuchote C. Formulation and characterization of topical anti-acne spot gel containing herbal extracts. MATEC Web Conf. 2018;237:1-6.

[45] Surini S, Sarah, Djajadisastra J. Formulation and in vitro penetration study of transfersomes gel containing gotu kola leaves extract (Centella asiatica L. Urban). J Young Pharm. 2018;10:27-31.

[46] Tønnesen HH, Karlsen J. Alginate in drug delivery systems. Drug Dev Ind Pharm. 2002;28:621-630.

[47] WHO. Guidelines for stability testing of pharmaceutical products containing well established drug substances in conventional dosage forms. World Health Organ Tech Rep Ser. 1996;863:65-80.

[48] Widyaningrum N, Fudholi A, Sudarsono, Setyowati EP. Stability of epigallocatechin gallate (EGCG) from green tea (Camellia sinensis) and its antibacterial activity against staphylococcus epidermidis ATCC 35984 and propionibacterium acnes ATCC 6919. Asian J Biol Sci. 2015;8:93-101.

[49] Wisuitiprot W, Somsiri A, Ingkaninan K, Waranuch N. In vitro human skin permeation and cutaneous metabolism of catechins from green tea extract and green tea extract-loaded chitosan microparticles. Int J Cosmet Sci. 2011;33: 572-579.

[50] Yanti Eff AR, Rahayu ST, Saraswati H, Munim A. Formulation and evaluation of sunscreen gels containing mangiferin isolated from Phaleria macrocarpa fruits. Int J Pharm Investig. 2019;9:141-145. 